\title{
Cytotoxic Factor in Dengue Haemorrhagic Fever
}

\author{
$\begin{array}{llll}\text { U.C. Chaturvedi R. Agarwal A. Misra R. Mukerjee } & \text { R. }\end{array}$ \\ S. Kapoor R. Nagar \\ Department of Microbiology, Lucknow, India
}

\section{Key Words}

Dengue haemorrhagic fever · Dengue virus . Cytotoxic factor $\cdot$ Cytokine

\begin{abstract}
Objective: Cytotoxic factor is a unique pathogenesis-associated cytokine that is produced in mice ( $\mathrm{mCF}$ ) and man ( $\mathrm{hCF}$ ) during dengue virus infection. This study was undertaken to investigate the prevalence of hCF and its relationship to the duration and severity of the illness, and to ascertain its role, if any, in the pathogenesis of dengue fever (DF) and dengue haemorrhagic fever (DHF). Methods: Peripheral venous blood was collected from the patients of various grades and on different days after the onset of clinical illness. Sera were collected from a total of 333 cases, and analysed for the presence of hCF by inhibition ELISA and dot blot tests. Result: The positivity for hCF was $100 \%$ in cases of DHF grades III and IV, while overall positivity was seen in 295 out of 333 (88\%) cases studied.
\end{abstract}

\begin{tabular}{ll}
\hline KARGER & (1) 1999 S. Karger AG, Basel \\
Fax +41 61 306 1234 34 & \\
$\begin{array}{l}\text { E-Mail karger@karger.ch } \\
\text { www.karger.com }\end{array}$ & $\begin{array}{l}\text { Accessible online at: } \\
\text { http://BioMedNet.com/karger }\end{array}$
\end{tabular}

Sera collected from the 1st to the 20th day of illness were positive for hCF. This was not seen at later periods. A majority of cases $(52 \%)$ were below 15 years of age and peak positivity of $96 \%$ was noticed in the agegroup of 11-15 years. The mean inhibition value of the ELISA was lowest (40 $\pm 8 \%$ ) in cases of DF and was highest (70 $\pm 10 \%)$ in DHF grade IV, and the peak titres were found on the first 4 days of the illness. Conclusion: The findings show the presence of hCF in the majority of cases. It is detectable up to the 20th day of illness and may suggest an association of higher levels of hCF with the onset and severity of the illness.

\section{Introduction}

During dengue virus infection a unique cytokine, the cytotoxic factor, is produced both in mice (mCF) and man (hCF) $[1,2]$. $\mathrm{mCF}$ is produced by L3T4-positive $\mathrm{T}$ cells 
and has a molecular weight of $43 \mathrm{kD}$ and an isoelectric point of $\mathrm{pH}$ 6.5. The amino-terminal sequence of mCF does not match so far (at the data base till December 1996) with any of the known proteins or cytokines [3; personal unpublished data]. We have shown the production of cytotoxic factor experimentally in mice $(\mathrm{mCF})$ only during dengue virus infection and not in others including an antigenically related Japanese encephalitis virus and unrelated polio, Coxsackie or ECHO viruses, a number of bacteria and mitogens [4]. $\mathrm{mCF}$ is similar to hCF in most of the properties including molecular mass, antigenicity, and reaction of their mRNA to the same probe in the Northern blot tests [5]. mCF induces an influx of $\mathrm{Ca}^{2+}$ into the target cells and selectively kills macrophages, helper $\mathrm{T}$ cells and the cells capable of liberating histamine by producing nitrite and respiratory burst, thus forming peroxynitrite [6-11]. $\mathrm{mCF}$ is a pathogenesis-related protein, capable of reproducing the pathological changes that are seen in the cases of dengue fever (DF)/dengue haemorrhagic fever (DHF), for example increased capillary permeability, cerebral oedema and blood leucocyte changes [3, 12-14].

The presence of hCF was shown in the sera of the cases of DF/DHF stored at AFRIMS, Bangkok and it was purified and characterized [2]. An extensive epidemic of DHF occurred in Northern India, including Delhi and Lucknow, during August to November 1996. This provided an opportunity to further extend the work by systematic studies to address many important questions regarding the role of hCF in cases of dengue, for example incidence of hCF-positive cases and the relationship of hCF with the age of the patients, duration and the severity of the illness. This report describes the presence of hCF in the majority of the cases of DF/DHF and may be related to the severity of the disease.

Cytotoxic Factor in Dengue

Haemorrhagic Fever

\section{Materials and Methods}

Cases

During the epidemic the diagnosis of dengue virus infection was suspected on the basis of the clinical presentation of a patient. All patients were subjected to Hess' test and the haematocrit and platelet counts were measured. The specific diagnosis for dengue virus infection was made by virus isolation and by the presence of virus-specific IgM. Only cases with established specific diagnosis were included in the present study. The patients were selected as they came (consecutively) to the hospital without any bias. The study was carried out on a total of 333 consecutive cases of DF/ DHF in whom diagnosis of dengue virus infection was established either by virus isolation (in 4 cases dengue type 2 virus was isolated) or by detection of the virusspecific IgM (in 229 patients) in the sera [data to be published]. The patients were either admitted to the Gandhi Memorial and Associated Hospitals, Lucknow, and Department of Pediatrics, All India Institute of Medical Sciences, New Delhi or were referred directly to this department. They were classified into those with DF or DHF grades I, II, III or IV according to the World Health Organization criteria [15]. For control, 50 normal healthy individuals (the first five decades of age had 10 individuals each), without history of a febrile or any other illnesses in the previous 3 months (hCF is not detectable after the 3rd week of illness), were included. The sera obtained from the cases and controls were screened for the presence of hCF by enzyme-linked immunosorbent assay (ELISA) and dot blot tests.

\section{Inhibition ELISA}

The principle of the test was that hCF present in the test sample bound with the antibody on the solid phase and inhibited subsequent binding of the protein A + HRP, thus indicating the presence of hCF. The technique was described elsewhere [16]. Briefly, a 1:5,000 dilution of hCF-specific antibody raised in mice [2] was put on the solid phase. The controls included were purified hCF as positive control and a heterologous protein as negative control. The OD from wells coated with antibody without addition of any antigen was considered as $100 \%$ binding of the protein A + HRP, while the wells without antibody coating yielded values for the blank. The percent inhibition was calculated as follows: $\%$ inhibition $=100-(\mathrm{OD}$ with test sample - OD of blank $) /(\mathrm{OD}$ without test sample - OD of blank) $\times 100$ [2]. The mean value +2 SD obtained from the 50 normal healthy controls was $30 \%$, and was considered as the 'cut-off' value.

Med Principles Pract 1999;8:26-31 27 
Table 1. Presence of hCF in the sera from cases with various grades of dengue illness

\begin{tabular}{lrrr}
\hline $\begin{array}{l}\text { Grades } \\
\text { of } \\
\text { illness }\end{array}$ & Patients & \multicolumn{3}{l}{ hCF-positive patients, n (\%) } \\
\cline { 3 - 4 } & & dot blot & ELISA \\
\hline DF & 127 & $100(79)$ & $102(80)$ \\
DHF I & 25 & $22(88)$ & $21(84)$ \\
DHF II & 139 & $111(80)$ & $111(80)$ \\
DHF III & 24 & $24(100)$ & $24(100)$ \\
DHF IV & 18 & $18(100)$ & $18(100)$ \\
\hline
\end{tabular}

Table 2. Presence of hCF in the sera collected on different days after the onset of dengue illness

\begin{tabular}{|c|c|c|c|}
\hline \multirow{2}{*}{$\begin{array}{l}\text { Days } \\
\text { of } \\
\text { illness }\end{array}$} & \multirow{2}{*}{$\begin{array}{l}\text { Patients } \\
\mathrm{n}\end{array}$} & \multicolumn{2}{|c|}{ hCF-positive patients, $\mathrm{n}(\%)$} \\
\hline & & dot blot & ELISA \\
\hline 1 & 6 & $6(100)$ & $6(100)$ \\
\hline 2 & 12 & $12(100)$ & $12(100)$ \\
\hline 3 & 25 & $24 \quad(96)$ & $25(100)$ \\
\hline 4 & 30 & $28 \quad(93)$ & $28 \quad(93)$ \\
\hline 5 & 60 & $47 \quad(78)$ & $48 \quad(80)$ \\
\hline 6 & 33 & $27 \quad(82)$ & $28 \quad(85)$ \\
\hline 7 & 40 & $29 \quad(73)$ & $34 \quad(85)$ \\
\hline 8 & 34 & $29 \quad(87)$ & $28 \quad(84)$ \\
\hline 9 & 18 & $14 \quad(81)$ & $15 \quad(83)$ \\
\hline 10 & 18 & $14 \quad(81)$ & $15(83)$ \\
\hline $11-15$ & 34 & $27 \quad(81)$ & $27(81)$ \\
\hline $16-20$ & 13 & $7 \quad(54)$ & $6(46)$ \\
\hline $21-26$ & 10 & $0 \quad(0)$ & $0 \quad(0)$ \\
\hline
\end{tabular}

Table 3. Presence of hCF in the sera from cases of various age-groups

\begin{tabular}{lll}
\hline Age-groups, years & Patients & Positive, \% \\
\hline $0-5$ & 64 & 89 \\
$6-10$ & 88 & 93 \\
$11-15$ & 23 & 96 \\
$16-20$ & 42 & 83 \\
$21-30$ & 64 & 87 \\
$31-40$ & 33 & 85 \\
41 and above & 19 & 84 \\
\hline
\end{tabular}

\section{Immunoblotting}

The technique described earlier was used $[2,17]$. Briefly, the sera were diluted tenfold in phosphatebuffered saline, $\mathrm{pH} 7.2$, and $100 \mu \mathrm{l}$ of it was blotted on nitrocellulose paper in Milliblot system (Millipore, USA). The blots were dried in air, blocked with $3 \%$ bovine serum albumin prepared in $0.1 \mathrm{M}$ Tris- $\mathrm{HCl}$, $\mathrm{pH}$ 7.5. After thorough washing with phosphate-buffered saline containing $0.05 \%$ Tween 20 , the blots were developed using anti-hCF antibody. For controls, the blots were treated with anti-dengue virus antibody or normal mouse sera in place of anti-hCF antisera.

\section{Results}

\section{Presence of $h C F$ in the Sera}

The findings of ELISA and dot blot tests in the sera from 333 cases of different grades of illness show that the two tests gave similar results, and 295 out of the 333 sera (88\%) were positive (table 1). All the sera from DHF grade III and IV cases were positive for hCF, while lowest positivity of $79 \%$ ( 100 out of 127 sera) was found in cases of DF grade. hCF was present from day 1 to 20 of the illness (table 2). At later periods (days 21-26) all the 10 sera were negative.

The data of the dot blot test for the agewise distribution of hCF-positive cases have been analysed (table 3) and show that 152 (46\%) patients were below 10 years of age and among them $140(92 \%)$ were positive for hCF. Only 1 child was below 1 year of age ( 8 months old). The highest positivity of $96 \%$ was found in children aged 11-15 years. Only 4 patients were above 51 years and the eldest was 57 years old. The findings showed no significant difference $(p>0.05)$ in the positivity for $\mathrm{hCF}$ in different age-groups. The study included 208 males (90\% positive) and 125 (92\% positive) females.

The data was further analysed in relation to the value of inhibition ELISA and the grade of illness to get a rough indication of relationship if any of the amount of hCF with the 
severity of illness. The findings summarized in figure 1 show that the mean value in the normal healthy controls was $20 \pm 5 \%$. Among the cases, the lowest value of $40 \pm 8 \%$ was seen in DF. It gradually increased in cases of DHF, the highest being $70 \pm 10 \%$ in DHF grade IV. A similar analysis in relation to the day of illness showed peak production of $\mathrm{hCF}$ during the first 4 days of the illness.

\section{Discussion}

The findings of the present study demonstrate the presence of $\mathrm{hCF}$ in $88 \%$ of the serum samples of the cases of DF and DHF. The presence of $\mathrm{hCF}$ in the serum was detected by ELISA and was confirmed by the dot blot tests in all the cases, and by the Western blot test in some of the cases by the technique described earlier [2].

In a study carried out on the sera of 220 cases of DF/DHF at Bangkok, the presence of $\mathrm{hCF}$ was shown in 98 (44\%) of them by the dot blot test [2] while the positivity was over $90 \%$ in the present study. In the Bangkok study, the sera used were stored for 2-3 years and had undergone repeated cycles of freezing and thawing and some of them had precipitates as well. In the present study, most of them were tested almost immediately after collection and a few of them had undergone one cycle of freeze and thaw. It is well known that repeated freezing and thawing is detrimental for any cytokine and therefore, fresh samples are the best. Further it has been shown that $\mathrm{mCF}$ aggregates on freezing [personal data]. The anti-mCF antibodies used in the earlier study [2] cross-react with hCF in ELISA, dot blot, Western blot and neutralization of the cytotoxicity tests [5, 17], but the anti-hCF antibody used in the present study may react more avidly giving better results. These points may explain the apparent dis-

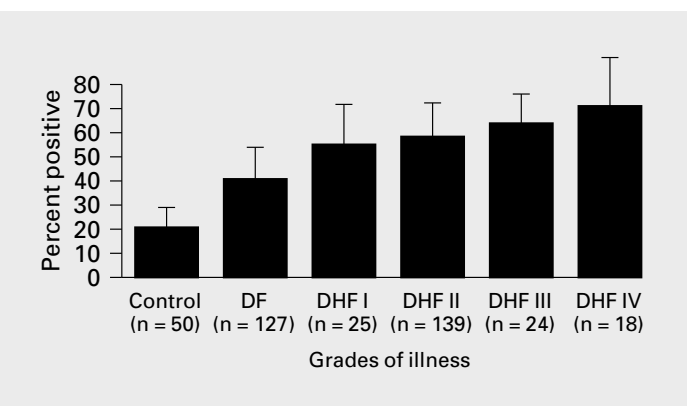

Fig. 1. Mean values of inhibition ELISA in the sera from controls and cases with various grades of illness. The bars over the column represent $\pm \mathrm{SD}$ and the figures in parentheses represent the total number of cases in each group.

crepancy in the results of the two studies. Of course the best would be the use of monoclonal antibody.

A confirmation of the present study is provided by the data presented elsewhere that show: (i) direct demonstration of the ex vivo production of hCF by CD4-positive T cells in peripheral blood mononuclear cells (PBMC) of the majority of the cases of DF/DHF; (ii) in situ demonstration of hCF in PBMC by fluorescent antibody test, and by (iii) demonstration of hCF-specific mRNA in the CD4-positive T cells of the PBMC by Northern hybridization [18, 19]. The normal healthy controls chosen for the present study represented the age-groups of the patients and 10 each were in each of the first five decades of life. The background of the findings so far that the cytotoxic factor is produced only during dengue virus infection [4] served to substantiate the absence of hCF in healthy individuals. We have looked for hCF-specific mRNA in the PBMC of these individuals and found it absent in all of them [18, 19].

Mice inoculated with dengue virus intraperitoneally produce cytotoxic factor $(\mathrm{mCF})$ which is detectable on the 5th-6th day after 
infection, reaches a peak on the 9th-11th day, and then gradually declines and is not detectable after the 16th day after infection [14]. This is supported by the present finding which showed the presence of hCF in the sera from the 1st day of illness to the 20th day with peak amounts of hCF during the first 4 days and then declined. In both mice and man, the peak levels of $\mathrm{mCF} / \mathrm{hCF}$ are associated with the appearance of the clinical illness and have a similar time course, considering the exposure of the body during the incubation period in man.

$\mathrm{mCF} / \mathrm{hCF}$ are unique cytokines that are seen only during dengue virus infection and not during infection with a closely related Flavivirus, the Japanese encephalitis virus, or unrelated agents like various enteroviruses, various mitogens, sheep erythrocytes, and bacteria, e.g. salmonella etc. Further, the Nterminal sequence of $\mathrm{mCF}$ has no homology with any of the known proteins or cytokines [3, 4; personal unpublished data]. Therefore, can hCF be an indicator of dengue virus infection and be used for the diagnosis of dengue? Difficulties encountered in rapid and reliable diagnosis of dengue have prompted us to initiate exploration of this possibility, using hCF purified from the sera of the cases or the recombinant hCF produced by its cDNA library prepared in $\lambda \mathrm{gt} 11$ and expressed in Escherichia coli to produce monoclonal antibody against hCF [personal unpublished data].

$\mathrm{mCF}$ is a pathogenesis-related cytokine and produces pathological lesions in mice that are seen in human cases of DHF, for example, increased capillary permeability [12], cerebral oedema [14] and alterations in the number and functions of leucocytes [13]. Purified hCF obtained from the cases of DHF produces increased capillary permeability and damages the blood-brain barrier on inoculation in mice [2]. hCF may have a role in the pathogenesis of DHF as indicated in the present study: (i) higher levels of hCF are associated with the onset of clinical illness; (ii) higher levels of $\mathrm{hCF}$ are found in cases with severe disease, the DHF grade IV as compared to mild DF $(p<0.001)$. Admittedly, the method used to assess the amount of hCF is not accurate, therefore, precise studies are needed to unequivocally establish this fact. The number of hCF-producing CD4-positive $\mathrm{T}$ cells in the blood of DHF cases may not reflect the amount of secreted hCF $[18,19]$. Therefore, the factors that control the levels of hCF need careful study.

\section{Acknowledgements}

We are grateful to Drs. A.K. Tripathi and K.L. Srivastava of K.G. Medical College, Lucknow and Prof. M.K. Bhan of the All India Institute of Medical Sciences, New Delhi for permission to study their cases. The study was carried out with the financial assistance of the Indian Council of Medical Research New Delhi. 


\section{References}

1 Chaturvedi UC, Bhargava A, Mathur A: Production of cytotoxic factor in the spleen of dengue virus infected mice. Immunology 1980; 40:665-671.

2 Mukerjee R, Chaturvedi UC, Vaughn DW, Kalayanarooj S: Purification and pathogenicity of the cytotoxic factor from the cases of dengue haemorrhagic fever. Curr Sci 1997;72:494-501

3 Khanna M, Chaturvedi UC: Purification and amino-terminal sequence of the dengue virus-induced cytotoxic factor. Int J Exp Pathol 1992;73:43-49.

4 Chaturvedi UC, Dhawan R, Mukerjee R: Immunosuppression and cytotoxicity of dengue infection in the mouse model; in Gubler DJ, Kuno $G$ (eds): Dengue and dengue haemorrhagic fever. Wallingford, Oxon, CAB International Press 1997, pp 289-309.

5 Mukerjee R, Chaturvedi UC: Cytokine antagonism by active vaccination. Curr Sci 1995;69:900-902.

6 Dhawan R, Chaturvedi UC, Masihi KN, Chaturvedi P, Mukherjee R: Depression of chemiluminescence during dengue virus infection of mice: Role of cytokines. Int J Exp Pathol 1993;74:455-461.
7 Khanna M, Chaturvedi UC, Dhawan R, Tekwani BL, Pandey VC: Presence of $\mathrm{Ca}^{2+}$ is obligatory for the cytotoxic activity of dengue virusinduced cytotoxic factor. Immunology 1990;72:73-78.

8 Misra A, Mukerjee R, Chaturvedi UC: Release of reactive oxygen intermediates by dengue virus-induced macrophage cytotoxin. Int $\mathbf{J}$ Exp Pathol 1996;77:237-242.

9 Misra A, Mukerjee R, Chaturvedi UC: Production of nitrite by dengue virus-induced cytotoxic factor. Clin Exp Immunol 1996;104:406-411.

10 Mukerjee R, Misra A, Chaturvedi UC: Dengue virus-induced cytotoxin releases nitrite by spleen cells. Int J Exp Pathol 1996;77:45-51.

11 Chaturvedi UC: Virus induced cytotoxic factor in AIDS and dengue. Immunol Today 1986;7:159.

12 Khanna M, Chaturvedi UC, Sharma MC, Pandey VC, Mathur A: Increased capillary permeability mediated by a dengue virus-induced lymphokine. Immunology 1990;69: 449-453.

13 Chaturvedi UC, Gulati L, Mathur A: Inhibition of E-rosette formation and phagocytosis by human blood leucocytes after treatment with the dengue virus-induced cytotoxic factor. Immunology 1982;45:679-684.
14 Chaturvedi UC, Dhawan R, Khanna M, Mathur A: Breakdown of the blood-brain barrier during dengue virus infection of mice. J Gen Virol 1991;72:859-866.

15 Nimmannitya S: Clinical manifestations of dengue/dengue haemorrhagic fever; in Thongcharoen P (ed): Dengue/Dengue Haemorrhagic Fever. New Delhi, WHO-SEARO, 1993, vol 22, pp 80-103.

16 Mukerjee R, Chaturvedi UC: ELISA for detection of dengue virus-induced cytokine and its antibody. Ind J Exp Biol 1997;35:225231.

17 Mukerjee R, Chaturvedi UC, Dhawan R: Dengue virus-induced cytotoxic factor: Production by peripheral blood leucocytes in vitro. Clin Exp Immunol 1995;102:262-267.

18 Agarwal R, Chaturvedi UC, Misra A, Mukerjee R, Kapoor S, Nagar R, Tandon R, Mathur A: Production of cytotoxic factor by peripheral blood mononuclear cells of the cases of dengue haemorrhagic fever. Clin Exp Immunol 1998;112:477-481.

19 Agarwal R, Chaturvedi UC, Misra A, Kapoor S, Nagar R, Tandon R: CD4 positive $T$ cells produce cytotoxic factor in cases of dengue haemorrhagic fever. Curr Sci 1998;74: 237-239. 